\title{
Rursus
}

Russus

Poiétique, réception et réécriture des textes antiques

$11 \mid 2017$

Nature et morale : sources, et postérité homilétique, des encyclopédies du XIIle siècle

\section{La réception des encyclopédies naturelles dans les sermons au XIIIe siècle. Quelques exemples}

The Reception of Natural Encyclopaedias in the Sermons of the thirteenth Century. Some examples

\section{Sophie Delmas}

\section{OpenEdition \\ Journals}

Édition électronique

URL : http://journals.openedition.org/rursus/1340

DOl : $10.4000 /$ rursus. 1340

ISSN : 1951-669X

Éditeur

Université Nice-Sophia Antipolis

\section{Référence électronique}

Sophie Delmas, "La réception des encyclopédies naturelles dans les sermons au XIIle siècle.

Quelques exemples », Rursus [En ligne], 11 | 2017, mis en ligne le 20 octobre 2017, consulté le 07 mai 2019. URL : http://journals.openedition.org/rursus/1340 ; DOI : 10.4000/rursus.1340

Ce document a été généré automatiquement le 7 mai 2019.

Rursus 


\section{La réception des encyclopédies naturelles dans les sermons au XIIIe siècle. Quelques exemples}

The Reception of Natural Encyclopaedias in the Sermons of the thirteenth

Century. Some examples

Sophie Delmas

1 Dans une étude récente, Peter Binkley soutient que les prédicateurs ne devaient guère avoir recours au savoir encyclopédique : après avoir examiné les thèmes de "dominion » et de " paix » et leur relation avec les grands encyclopédistes du XIII ${ }^{e}$ siècle (Thomas de Cantimpré, Barthélemy l'Anglais et Vincent de Beauvais), il défend l'idée selon laquelle ces œuvres, qui devaient servir aux clercs dans leurs sermons et dans leurs commentaires de l'Ecriture, ne pouvaient guère satisfaire leurs auditeurs. En effet, les encyclopédistes décrivaient un monde naturel caractérisé par la paix et l'ordre, alors que les prédicateurs étaient confrontés à un monde humain « réel » caractérisé par le péché et le conflit. Son article s'achève par ce jugement sans appel: «if the Summa predicantium and similar preaching aids are not encyclopaedias, it is because Bromyard and his colleagues thought the encyclopaedists had got the world upsidedown and were trying to put it right ${ }^{1}$ ».

2 Si ce point de vue peut éventuellement se comprendre dans le domaine politique, il ne peut guère être étendu à tous les champs du savoir. Ainsi, plusieurs prédicateurs médiévaux ont indéniablement utilisé les encyclopédies pour construire leurs sermons : dans un de ses articles, Louis Jacques Bataillon soulignait que, parmi les instruments de travail des intellectuels médiévaux, il ne fallait «oublier ni les florilèges d'auteurs classiques ou philosophiques, ni les diverses encyclopédies. Bestiaires, lapidaires ont été souvent consultés (...) : l'aigle, le cerf, la lionne et tant d'autres animaux qui hantent nos sermons, ne viennent ni des forêts, ni des jardins du roi, mais bien de ces ouvrages ${ }^{2}$.

Cependant, si les compilateurs de recueils d'exempla ont parfois explicitement mentionné les emprunts aux encyclopédies, ce n'est que rarement le cas des prédicateurs qui y ont pourtant largement puisé3. Malgré cette difficulté, j'essaierai ici de montrer comment les 
sermons ont été les premiers destinataires de la documentation propre à l'encyclopédisme médiéval. Dans ce but, j'étudierai d'abord dans quelle mesure les encyclopédies sont perçues comme un instrument de travail des prédicateurs. Puis, pour limiter le propos, je tenterai de voir comment quelques prédicateurs mendiants ont pu utiliser les savoirs encyclopédiques liés plus particulièrement au monde animal ou végétal.

\section{Les encyclopédies, manuels des prédicateurs ?}

4 L'utilité des savoirs - et plus particulièrement des propriétés des choses - pour la prédication est clairement exprimée par les auteurs mêmes des encyclopédies qui ont expliqué cet usage dans les prologues et l'ont favorisé par la mise en place de moralisations dans les notices mêmes de leurs œuvres. De leur côté, les prédicateurs ont cherché à utiliser ces connaissances dans leurs discours, comme en témoignent les manuels pour prédicateurs, les artes predicandi et les développements des sermons.

\section{L'enseignement des prologues des encyclopédies}

5 D'une manière générale, les prologues des encyclopédies témoignent de la volonté de leur auteur d'être "utiles à la postérité » (ob utilitatem posteritatis) - comme l'a récemment souligné Isabelle Draelants - et plus particulièrement aux prédicateurs ${ }^{4}$. Certains auteurs restent parfois vagues : Barthélemy l'Anglais, dans le prologue général du De proprietatibus rerum, se contente d'expliquer qu'il compile cet ouvrage qui sera utile «à lui et peut-être à d'autres » (utile mihi et forsitan aliis) $)^{5}$. En revanche, le prologue général du Speculum maius de Vincent de Beauvais est davantage explicite : selon l'auteur, son œuvre peut être utile pour prêcher, lire (commenter), pour disputer et même d'une manière générale pour aborder n'importe quel sujet: "Car j'en suis en effet certain - et j'ai confiance dans le Seigneur -, cette œuvre sera d'une grande utilité (...), non seulement afin de connaître Dieu en lui-même et à travers ses créatures visibles et invisibles, et par là l'aimer (...), mais encore afin de prêcher, lire, disputer, résoudre les questions et d'une façon générale de traiter de tout genre de matière dans chacun des arts $»^{6}$. De même, dans le prologue du Liber de natura rerum, Thomas de Cantimpré est clair sur son souhait de fournir un instrument de travail pour les prédicateurs : selon lui, il est nécessaire de bien connaître les créatures pour adorer leur créateur7. Il ajoute même que, "alors que les discours rebattus sur les Écritures n'émeuvent plus l'auditoire, il pourra du moins attirer l'oreille des paresseux en parlant de quelque chose de nouveau $»^{8}$.

\section{Les moralisations des encyclopédies}

6 Cette volonté de servir aux prédicateurs apparaît plus nettement encore dans les moralisations proposées par certaines encyclopédies : dans certains cas, les moralisations d'origine sont placées dans les marges des manuscrits et font partie intégrante de l'œuvre. Ainsi, plusieurs auteurs ont utilisé ce procédé bien utile pour la composition des sermons.

7 Au tournant des $\mathrm{XII}^{\mathrm{e}}$ et $\mathrm{XIII}^{\mathrm{e}}$ siècles, dans le De naturis rerum, Alexandre Neckam propose, à l'usage direct du prédicateur, soit des «adaptations» (adaptatio), soit des «instructions morales » (instructio moralis), signalées dans les marges des manuscrits : J. Berlioz et M.-A. 
Polo de Beaulieu ont ainsi trouvé un parallèle entre les mœurs de l'alouette et sa moralisation décrite dans cette encyclopédie et un passage d'un sermon évoqué par Nicole Bériou ${ }^{10}$.

Dans le Liber de natura rerum de Thomas de Cantimpré (vers 1240), J. Francisco Talavera Esteso a relevé de nombreux passages dans lesquels l'auteur propose une grande variété de développements symboliques ou religieux qui pourront servir de manière très concrète au prédicateur ${ }^{11}$. Ces développements religieux peuvent avoir une tonalité ascétique ou morale. Ils sont introduits de différentes manières : on peut les trouver sous forme d'explication (avec les verbes signare ou significare) ou encore sous forme de questions rhétoriques. Un passage est présenté d'une façon plus claire encore puisque l'auteur explique qu'il propose une interprétation morale du phénix (Item de fenice moraliter). Ces moralisations permettent à l'auteur de dénoncer certains défauts tels que l'hypocrisie ou l'orgueil.

9 Cette réponse de l'encyclopédie aux besoins de la prédication est encore plus manifeste dans le De proprietatibus rerum de Barthélemy l'Anglais (vers 1234-1245). Dans de nombreux manuscrits, le texte est accompagné de nombreuses notes marginales du type nota de qui indiquent brièvement le sens allégorique et moral à donner aux réalités évoquées dans le corps du texte. Heinz Meier a démontré que ces notes, loin d'être des additions de copistes, étaient, dès l'origine, solidaires du texte ${ }^{12}$. Ensuite, l'œuvre du franciscain a été à la fois remaniée et moralisée, comme dans le Liber septiformis de moralibus rerum, rédigé entre 1281 et 1291 par le franciscain Marc d'Orvieto. Connue par au moins dix-huit manuscrits, cette œuvre prend pour point de départ les notes marginales et en propose un commentaire plus dense comprenant des commentaires moraux et des citations bibliques, ce qui le rendait très utile pour la prédication ${ }^{13}$.

10 En s'appuyant sur les prologues et les dédicaces, Christel Meier propose de distinguer huit sortes d'encyclopédies selon leur fonction. La quatrième d'entre elles rassemble « les encyclopédies des prédicateurs » (" preachers' encyclopaedias »). Il s'agit précisément de ces encyclopédies moralisées qui sont de plus en plus diffusées à la fin du Moyen Âge comme le montrent une centaine de manuscrits subsistant. Ces encyclopédies insistent sur les choses, les signifiants (significantia) et leurs signifié (significata). Les exemples retenus par Christel Meier sont les œuvres de Marc d'Orvieto, Henri de Schüttenhofen, Jean de San Gimignano et le Reductium morale de Petrus Berchorius (Pierre Bersuire) ${ }^{14}$.

11 Ainsi, si les encyclopédistes avaient conscience de pouvoir être utiles aux prédicateurs, comme le montrent leurs prologues, et se préoccupaient de leur fournir des outils faciles à manier, comme le prouvent les moralisations, les prédicateurs, eux, se montrent plus discrets lorsqu'il s'agit de puiser dans le savoir encyclopédique.

\section{L'enseignement des artes predicandi}

12 Cet intérêt pour les encyclopédies peut d'abord être recherché dans les manuels pour prédicateurs. Les artes predicandi se développent à partir de la fin du $\mathrm{XII}^{\mathrm{e}}$ siècle et quelques-uns ont été édités. Certains d'entre eux ont en outre été analysés par Franco Morenzoni ${ }^{15}$. À ma connaissance, ces textes ne font pas directement référence à des auteurs ou à des ouvrages encyclopédiques. En revanche, un premier dépouillement m'a permis de répertorier quelques passages faisant indirectement référence à l'utilisation du savoir encyclopédique. 

Morenzoni, reprennent cette idée, à commencer par Thomas de Chobham qui, vers 1220, conseille dès le prologue de la Summa de arte predicandi d'utiliser les propriétés des animaux et de toutes les choses ${ }^{17}$. Ces éléments, insiste-t-il, sont importants pour toucher les cœurs des auditeurs. Il range ce procédé parmi les narrationes: en effet, le développement du sermon est à ses yeux constitué de récits, soit des récits historiques qui évoquent des faits qui ont réellement eu lieu, soit des récits englobant les paraboles, les fables et les moralisations des animaux. Thomas propose du reste un exemple concret, celui des similitudes utilisées pour désigner le Seigneur. Il explique que ce dernier peut être comparé au lion : en effet, le Seigneur, comme le lion, domine toutes les créatures. Il poursuit en précisant que le courage du lion est dans sa poitrine, sa solidité dans sa tête ; que les lions captifs craignent le bruit des roues et par-dessus tout redoutent le coq blanc ${ }^{18}$. Ces explications sont celles des bestiaires et on les lit par exemple chez Barthélemy l'Anglais ${ }^{19}$.

$\mathrm{Au}$ XIII ${ }^{\mathrm{e}}$ siècle toujours, le chanoine anglais Richard de Thetford énumère huit manières permettant de développer un sermon : le sixième mode consiste à expliquer l'Écriture en faisant appel aux propriétés réelles des choses signifiées ${ }^{20}$.

16 Au siècle suivant, dans son De arte predicandi, le prédicateur dominicain Jacques de Fusignano ( $†$ en 1333) explique que le prédicateur peut développer son sermon «à l'aide des propriétés des choses lorsque dans un thème, il faut exposer certaines choses naturelles ou produites par l'homme dont les propriétés peuvent être adaptées à la louange des saints dont il est question dans la prédication ou aux bonnes mœurs ». Cependant, il ajoute qu'il faut nécessairement soumettre ces développements scientifiques à un objectif d'édification morale et religieuse ${ }^{21}$. De même, on peut trouver une allusion dans le De modo componendi sermones du maitre en théologie d'Oxford Thomas Walleys (†1349) qui suggère, parmi les procédés de développement, d'expliquer les propriétés d'une chose mentionnée dans une autorité : il ajoute que ce procédé suppose une bonne connaissance des propriétés des choses.

17 Il serait inutile de multiplier les exemples. Il est évident que ces passages, extraits des Artes predicandi et faisant allusion aux propriétés des choses, font indéniablement penser aux encyclopédies, et même plus précisément au titre de celle de Barthélemy l'Anglais ${ }^{22}$. Ce dernier, comme Vincent de Beauvais, insiste sur la nécessité d'étudier ces propriétés ${ }^{23}$. Ces différents passages ont probablement une source commune, un passage du De doctrina christiana de saint Augustin selon lequel il est légitime d'étudier les propriétés et la nature des choses : ces dernières permettent d'éclairer la création du monde et son message transmis par les expressions figurées des Écritures ${ }^{24}$. 


\section{La transmission du savoir encyclopédique dans les sermons : le cas du monde animal et végétal}

Les encyclopédies et leurs auteurs, à ma connaissance, sont rarement cités nommément dans les sermons. En revanche, un examen attentif des sources pastorales témoigne de cet usage, particulièrement significatif dans le cas de la faune et la flore.

\section{Des références implicites dans les sermons reportés ${ }^{25}$}

Dans la majorité des cas, l'utilisation des encyclopédies reste implicite, ce qui rend parfois difficile son identification.

Quelques formules peuvent attirer l'attention et laisser supposer l'utilisation du savoir encyclopédique. À plusieurs reprises, le franciscain Eustache d'Arras auquel j'ai consacré un livre évoque les propriétés des choses : il parle des propriétés des montagnes (quatuor proprietates uidemus in monte) dans le sermon 9 ; des propriétés naturelles du cerf (ceruus enim habet uelocitatem in cursu, amplitudinem et altitudinem in saltu, uehementiam in appetitu, et durabilitatem in statu. Iste sunt quatuor proprietates naturales siue condiciones cerui) dans le sermon 10; des propriétés de la colombe dans le sermon 11 (Ionathas fuit beatus Bartholomeus per uite innocentiam, ad quam quatuor predicta exiguntur que per columbe proprietates designantur) ; ou des propriétés du figuier (Habet autem ficulnea cum fructu suo octo proprietates anime et uite hominis sentientis et procurantis sibi consolationes celestes conuenientes) dans le sermon $16^{26}$. Ces propriétés sont importantes: elles ne sont pas seulement un exemple proposé dans la prédication du maitre, elles sont structurantes, c'est-à-dire que le prédicateur construit le plan de son développement en s'appuyant sur les propriétés énumérées.

21 Ainsi, Eustache d'Arras choisit de développer son sermon 16 autour des huit caractéristiques du figuier : celles-ci sont en partie empruntées au De proprietatibus rerum de Barthélemy l'Anglais, sans qu'il soit nommément cité. À chacune mention des propriétés de l'arbre, Eustache associe une interprétation spirituelle visant à montrer que les propriétés sont aussi celles de la consolation divine.

Le thème du sermon est issu de l'Evangile de Luc: «Voyez le figuier et les autres arbres. Dès qu'ils bourgeonnent, vous comprenez de vous-mêmes, en les regardant, que désormais l'été est proche. Ainsi, lorsque vous verrez cela arriver, comprenez que le Royaume de Dieu est proche» (Lc. 21, 29-31). À partir de ce thème biblique, Eustache d'Arras explique que le Seigneur a donné aux âmes un remède qui permet de faire face au danger. Le figuier et son fruit possèdent huit caractéristiques qui procurent à l'homme des consolations divines. La première est que le figuier, plus que tous les autres arbres, est fécond; de même la consolation divine rend l'âme plus féconde en biens spirituels. La seconde est que, comparée aux autres fruits, la figue est beaucoup plus douce; de même, la consolation divine rend l'âme plus douce. La troisième est qu'elle réconforte plus que les autres fruits; de même, la consolation divine réconforte mieux que n'importe qui. La quatrième est que les figues retendent les rides des vieillards qui en ont mangé; de même, la consolation divine enlève les rides du péché. La cinquième est que les taureaux les plus féroces s'adoucissent une fois attachés à un figuier; de même, la consolation divine adoucit les tyrans et les empereurs et les impies. Eustache emprunte alors un 
passage du début de l'Épitre aux Galates dans lequel Paul explique les persécutions qu'il a menées, avant d'avoir sa révélation intérieure qui le conduit à devenir un apôtre parmi les païens. La sixième est que le lait du figuier a la force de « coagulation » et a la vertu de coagulation pour faire des fromages; de même, la consolation divine permet à l'âme de se rassembler et de s'unir à Dieu. La septième est que les figuiers sont par nature plutôt situés sous les vents du Midi que sous l'Aquilon. De même, il faut accueillir l'inspiration de l'Esprit saint et repousser les tempêtes du diable, du monde et de la chair. La huitième est que les figues permettent de soigner de nombreuses maladies; de même la consolation divine efface toutes les infirmités de l'âme.

Le tableau suivant permet de mieux comprendre l'utilisation des données encyclopédiques :

\begin{tabular}{|c|c|}
\hline $\begin{array}{l}\text { Bartholomaeus Anglicus, De } \\
\text { proprietatibus rerum. Volume VI: Liber } \\
\text { XVII, éd. Iolanda Ventura, Turnhout, } \\
\text { Brepols (De diversis artibus, 79, n. s. 42), } \\
2007 \text {, p. } 91-94\end{array}$ & Eustache d'Arras, sermon 16 \\
\hline $\begin{array}{l}\text { Ficus est arbor a fecunditate dicta; aliis } \\
\text { enim arboribus feracior est, nam terque } \\
\text { quaterque per singulos annos fructum } \\
\text { gignit, atque altero maturescit aboritur } \\
\text { alter statim. } \\
\text { [...] inter fructus carica est dulcior, et est } \\
\text { utilis in cibo et in medicina; multum enim } \\
\text { nutrit et impinguat. } \\
\text { [...] ante tempora vero Pythagore alebantur } \\
\text { ficubus Galathe, alias Athelete, antequam } \\
\text { idem cos ad carnis usum, in quo fortior est } \\
\text { cibus, transtulisset. } \\
\text { [...] a senibus autem in cibo sumpte ficus } \\
\text { rugas eorum distendere feruntur. } \\
\text { [...] tauros quoque ferocissimos ad fici } \\
\text { arbores colligatos dicunt repente } \\
\text { mansuescere. } \\
\text { [...] fici lac vim habet coaguli ad faciendos } \\
\text { caseos. } \\
\text { [...] in aquilonati parte minus proficiunt fici } \\
\text { et plus arescunt, quia earum humor lacteus } \\
\text { facillime consumitur. }\end{array}$ & $\begin{array}{l}\text { Habet autem ficulnea cum fructu suo octo } \\
\text { proprietates anime et uite hominis sentientis et } \\
\text { procurantis sibi consolationes celestes } \\
\text { conuenientes. } \\
\text { Prima est quia ficus omnibus aliis arboribus est } \\
\text { fructu fecondior siue feracior. Nam ter quinque in } \\
\text { anno fructificat et, uno fructu nato, alter est in } \\
\text { nascendo et, uno maturo, alter est in } \\
\text { maturescendo (...). } \\
\text { Secunda est quia omnibus aliis fructibus est } \\
\text { dulcior et pinguior [...]. } \\
\text { Tercia est quia maioris ceteris fructibus est } \\
\text { confortacionis. In cuius signum, ante tempora } \\
\text { Pictagore, athlete alebantur ficibus sicut modo } \\
\text { carnibus [...]. } \\
\text { Quarta est quia ficus a senibus in cibo sumpte } \\
\text { rugas eorum contendunt [...]. } \\
\text { Quinta est quia tauri ferocissimi ad fici arborem } \\
\text { alligati dicuntur mansuescere [...]. } \\
\text { Sexta est quia lac fici uim coagulari (sic) habet ad } \\
\text { faciendos caseos [...]. } \\
\text { Septima est quia arbor fici naturalius situatur } \\
\text { contra uentos australes quam contra aquilonem. } \\
\text { Nam lacteus eius humor facile consumitur ab } \\
\text { aquilonaribus uentis [...]. } \\
\text { Octaua est quia contra multas egritudines ualent } \\
\text { ficus : nam pectus mundificant, tussim sedant, } \\
\text { faucium tumorem mitigant et huiusmodi multa } \\
\text { faciunt. }\end{array}$ \\
\hline
\end{tabular}


Cet exemple significatif dans la prédication d'Eustache d'Arras m'a incitée à chercher d'autres passages témoignant de l'utilisation du savoir encyclopédique. Cependant, cette recherche n'a pas donné beaucoup de résultats. Les passages sur le cochon (sermons 7, 22), sur l'âne (sermon 8), sur l'aigle (sermon 9), sur la mouche (sermons 14, 25), sur le cheval (sermon 10) ou la colombe (sermon 11) ne se retrouvent guère de façon significative dans les encyclopédies bien éditées jusqu'ici. Il est probable que certaines de ces images animalières proviennent d'autres sources telles que les œuvres exégétiques des Pères de l'Eglise, la glose ${ }^{27}$ et probablement les recueils de propriété dont E. Kuhry a récemment montré le rôle d'instrument de travail pour les prédicateurs ${ }^{28}$. C'est le cas notamment pour la colombe : Eustache d'Arras explique que la colombe est auis carens felle / columba semper eligit grana meliora et illis uescitur/ frequenter habet gemitum pro cantu. Or ces éléments se retrouvent dans la glose marginale sur le Cantique des Cantiques $(1,14)$ : columba felle caret, rostro non ledit, in cauernis petrarum nidificat, alienos pullos nutrit, iuxta fluenta manet, meliora grana eligit, gemitum pro cantu reddit, gregatim uolat, alis se defendit, uisum recuperat ${ }^{29}$. Ces propriétés se lisent aussi dans des exempla présents dans les Homiliae de infantia Servatoris de Césaire d'Heisterbach: il y est expliqué que la colombe est dépourvue de fiel, nourrit les petits des autres, ne mange pas de vers mais choisit les meilleurs grains et vole en groupe ${ }^{30}$. D'autres enfin se lisent directement dans la Bible, par exemple la description de l'aigle faisant son nid dans les hauteurs (Job 39, 27 : Numquid ad praeceptum tuum eleuabitur aquila, et in arduis ponet nidum suum). Cette volonté de construire le nid en altitude est associé, dans certains récits exmplaires, à la volonté du rapace de se rapprocher du soleil ${ }^{31}$.

\section{Les images du monde animal chez Eustache d'Arras}

\begin{tabular}{|l|l|l|}
\hline $\begin{array}{l}\text { Animal } \\
\text { mentionné }\end{array}$ & $\begin{array}{l}\text { Numéro } \\
\mathbf{d u} \\
\text { sermon }\end{array}$ & Caractéristiques de l'animal \\
\hline $\begin{array}{l}\text { Le cochon } \\
\text { (porcus) }\end{array}$ & $\begin{array}{l}\text { Sermon 7 } \\
\text { Sermon 22 }\end{array}$ & $\begin{array}{l}\text { - Sunt etiam tales sicut porcus qui comedit leglan neque umquam respicit } \\
\text { - Tales sunt porcis similes qui dum uiuunt nihil boni faciunt, immo } \\
\text { animalia immunda et nociua sunt in hospitio. Sed quando interficiuntur, } \\
\text { multa bona inde exeunt. }\end{array}$ \\
\hline $\begin{array}{l}\text { L'âne } \\
\text { (asinus) }\end{array}$ & Sermon 8 & $\begin{array}{l}\text { Sicut scitis, qui uult probare fortitudinem asini, probat eam per } \\
\text { appositionem super eum magni ponderis. Si uere non possit ferre magnum } \\
\text { pondus, probatum est quod sit debilis. }\end{array}$ \\
\hline Aigle (aquila) & Sermon 9 & $\begin{array}{l}\text { Aquila ualde alte uolat, aquila Virgo Maria, recte aquila, genere nobilior, } \\
\text { uisu perspicatior et uolatu fortior. Talis quidem fuit Virgo Maria: hec est } \\
\text { aquila cuius nidus, id est quies, posita est in arduis }\end{array}$ \\
\hline
\end{tabular}




\begin{tabular}{|l|l|l|}
\hline $\begin{array}{l}\text { Mouche } \\
\text { (musca) }\end{array}$ & Sermon 14 & $\begin{array}{l}\text { - Scitis quod musca residet ubi uidit crassa et intoxicat ea. Similiter } \\
\text { dyabolus mittit temptationem suam ubi uidet bona ut illa impediat. Qui } \\
\text { muscam comedit, uomit totum quod comedit. } \\
\text { - Male autem cogitationes sunt sicut musce dyaboli, musce que inquietant } \\
\text { multum et infestant homines, incident scutellis et ciphis et quandoque } \\
\text { permittunt se cadere in scutella }\end{array}$ \\
\hline $\begin{array}{l}\text { Cheval } \\
\text { (equus) }\end{array}$ & Sermon 10 & $\begin{array}{l}\text { Infirmitates exteriores sunt sicut calcaria Dei quibus stimulas ad } \\
\text { operationes bonas quando equus stimulatur calcaribus, ita quod exit } \\
\text { sanguis de carne, statim ponit se in cursum. }\end{array}$ \\
\hline $\begin{array}{l}\text { Oiseaux } \\
\text { (aves) }\end{array}$ & Sermon 9 & $\begin{array}{l}\text { Vos uidetis quod aucupes ponit laqueos in terra ad capiendum aues et } \\
\text { quando uolat auis inferius prope terram cito capitur. Sed si semper uolaret } \\
\text { alte, numquam caperetur }\end{array}$ \\
\hline $\begin{array}{l}\text { Colombe } \\
\text { (columba) }\end{array}$ & Sermon 11 & $\begin{array}{l}\text { Quatuor exiguntur que in columba designantur, scilicet pietas affectionis } \\
\text { rectitudo intentionis, fecunditas operationis, frequentia deuote orationis : } \\
\text { columba est auis carens felle / columba semper eligit grana meliora et illis } \\
\text { uescitur/ frequenter habet gemitum pro cantu. }\end{array}$ \\
\hline
\end{tabular}

La raison est peut-être à chercher dans la nature des textes utilisés : la trentaine de sermons d'Eustache d'Arras conservés sont des sermons reportés, c'est-à-dire qu'ils ont été pris en note à l'audition. Les auditeurs n'ont peut-être pas reproduit avec exactitude les passages concernant la nature, les propriétés des choses ou tout simplement ne les ont pas retenus dans leurs annotations. Ce n'est donc sans doute pas dans les sermons reportés qu'il faut rechercher les traces précises du savoir encyclopédique, mais plutôt dans les "sermons rédigés ", pour reprendre une expression de Louis Jacques Bataillon, c'est-à-dire des sermons souvent qualifiés de "modèles » : ils ont été mis par écrit par leur auteur ou par des clercs dans l'idée de fournir des éléments réutilisables ${ }^{32}$.

\section{Des références rares, mais précises dans les sermons rédigés ou « modèles »}

C'est pour cette raison qu'il me semble intéressant de poursuivre l'enquête dans cette autre catégorie de sermons, les sermons rédigés ou " modèles " ${ }^{33}$. Je m'appuierai sur deux recueils de sermons modèles, celui du frère mineur du XIII ${ }^{e}$ siècle Guibert de Tournai et celui du frère prêcheur du XIV ${ }^{\mathrm{e}}$ siècle Nicoluccio di Ascoli.

Guibert de Tournai est connu pour avoir été maître régent au studium de Paris après Bonaventure, dans la seconde moitié du XIII ${ }^{e}$ siècle. Proche du pape comme du roi de France, il est connu pour son éloquence. Il a laissé une œuvre variée, notamment des recueils de sermons. L'édition des sermons ad status du franciscain Guibert de Tournai sera bientôt accessible à tous puisque Marjorie Burghart vient de les étudier dans sa thèse de doctorat, récemment soutenue ${ }^{34}$. Dans son travail, elle a noté l'importance du bestiaire de Guibert: elle a relevé quarante et un passages dans lesquels sont évoqués des comportements de pas moins de trente-huit espèces ou types d'animaux. Ces animaux sont très variés : « les espèces vont des animaux les plus insignifiants (mouche, sangsue, abeilles, fourmis) aux plus imposants (l'éléphant ou le marsouin - porcus marinus), en 
passant par les espèces familières, domestiques (âne, cheval, chien, taureau) ou sauvages (blaireau, castor, cerf, grenouille, hérisson, loup, marmotte, ours, poissons, renard, sanglier), sans oublier quelques espèces «exotiques " (lion, panthère, guenon) voire imaginaires (« mirmicoleon » et dragon). Les oiseaux sont également bien représentés, avec dix espèces citées ${ }^{35} \%$.

Ainsi, Guibert explique que le "mirmicoleon » est un petit animal qui se cache dans la poussière, attaque les fourmis et mange celles qui ne peuvent résister; s'il se montre envers elles comme un lion, il est pour les oiseaux et les autres animaux comme une fourmi qu'ils mangent. Cette explication se lit par exemple chez Thomas de Cantimpré (IX, De vermibus, c. 22 De formicoleon) dans un passage emprunté à " Adelinus ", puis chez Vincent de Beauvais qui emprunte à son confrère dominicain la notice ${ }^{36}$.

Un autre exemple est celui des fourmis, à propos desquels Guibert renvoie aux « livres d'histoire naturelle »: le maitre franciscain souligne qu'elles n'ont pas de guide, qu'elles ne voient pas clairement. C'est pourquoi il leur a été donné en guise de canne une sorte d'ajout dans leur partie antérieure, avec laquelle elles se dirigent en testant le chemin.

Dans les sermons ad status, Guibert de Tournai n'indique jamais la source de ses informations. Cependant, dans ce sermon adressé aux puissants et aux soldats (ad potentes et milites), il dit avoir puisé les caractéristiques des fourmis dans des ouvrages de science naturelle (libri naturales) ${ }^{37}$. Ce type de référence se retrouve chez d'autres prédicateurs. Ainsi, chez le prédicateur cistercien Jean de Villers, on trouve également l'expression dicunt naturales. De même, dans de nombreux sermons de la fin du Moyen Âge, les prédicateurs témoignent d'emprunts livresques par les mots : dicunt naturales ou ponunt phisicis ${ }^{38}$.

31 Moins d'un siècle plus tard, le dominicain Nicoluccio di Ascoli, étudié par Xavier Masson, est lui aussi particulièrement attentif au monde animal. Surtout, contrairement à Guibert de Tournai, il va se montrer beaucoup plus précis sur ses sources d'information.

Ce prédicateur dominicain a rédigé vers 1340 un recueil de sermons destiné aux futurs frères de son ordre. Natif de la cité d'Ascoli Piceno dans les Marches italiennes, Nicoluccio a fréquenté l'Université de Bologne dans les années 1320, puis est devenu prieur aux couvents de San Pietro Martire et de San Andrea au cours des deux décennies suivantes.

Dans son sermon 30, il évoque ainsi le fait que si l'amandier (amigdalus) devient amer, il peut être conservé et passe alors de l'amertume à la douceur. L'arbre est alors troué et au milieu est placé le fumier d'un autre arbre, le pin, ce qui fait disparaitre l'amertume. Le prédicateur attribue ce passage à Alexandre Neckam (Alexander Nequam dicit), chez qui nous ne l'avons pas trouvé dans le De naturis rerum, mais il se trouve en fait chez Vincent de Beauvais ${ }^{39}$.

Des références précises sont également données par Nicoluccio di Ascoli à propos des animaux : à plusieurs reprises, il cite un ouvrage intitulé le De naturis rerum, mais selon Xavier Masson, il ne correspond à aucune des encyclopédies les plus connues (Thomas de Cantimpré, Alexandre Neckam, Vincent de Beauvais ou Barthélemy l'Anglais ${ }^{40}$. C'est le cas pour l'éléphant : «Selon ce qui est dit dans le livre De naturis rerum, quand ils doivent s'avancer dans les chemins difficiles, les plus petits éléphants marchent en tête et les plus anciens et les plus grands s'avancent sur leurs traces. La raison en est que l'éléphant, à cause de la mollesse de son corps et de sa grande force laisse des traces très profondes. Il est donc très dangereux pour les autres animaux plus petits de suivre leurs traces car leurs pieds sont blessés et cassés facilement. »C'est aussi le cas pour le serpent : « Comme 
il est dit dans le livre De naturis rerum, le serpent, quand il est usé par l'âge et qu'il veut changer de peau, fait abstinence pendant plusieurs jours pour s'éloigner de la nourriture et pour que sa peau soit plus facilement relâchée ; ensuite il se place entre les arêtes et les trous des pierres, y laisse sa vieille peau et endosse la nouvelle. Spirituellement, le Christ se considère comme un serpent dans le désert en disant : « Comme Moïse éleva le serpent dans le désert $(J e a n 3,14)[. .$.$] . Il en va de même pour nous après que nous avons jeûné$ pendant quarante jours, nous laissons notre vieille peau du péché dans le trou du sépulcre. » Ces deux passages semblent cependant avoir beaucoup de similitudes avec le Liber de naturis rerum de Thomas de Cantimpréet ${ }^{41}$

Il est donc parfois bien difficile pour l'historien d'identifier les sources des prédicateurs. Franco Morenzoni qui a étudié les animaux exemplaires dans les recueils de distinctiones s'est heurté à la même difficultét2 ${ }^{42}$ En effet, dans son recueil de Distinctiones, le dominicain Nicolas de Biard cite explicitement un passage qu'il attribue à un livre sur les natures des animaux. Il y décrit la méthode utilisée par l'onagre pour échapper aux chiens qui le poursuivent : nota de natura onagri. Dicitur enim in libro de naturalium animalium $\left(s i c^{43}\right)$ quod onager insecutus a canibus consolidat cibum in stomacho et post emittit fimum canibus qui ibi remanent et ita evadit. Sic divites, temporalia que quasi fimus sunt, dando, ea prelatis et predicatoribus ne eos arguant. Ce passage n'est pas dans Barthélemy l'Anglais, mais un extrait similaire se lit à nouveau dans le Liber de naturis rerum de Thomas de Cantimpré ( onager ex naturali industria, quando eum persecuuntur canes, emittit stercus suum odoriferum canibus. Circa quod detentis canibus, eos illudit et evadit et fugit ad tutiora ${ }^{44}$ ). L'usage probable du Liber de natura rerum me semble d'autant plus convaincant dans la mesure où cette œuvre a d'abord circulé de façon anonyme ou avec des noms d'emprunt, ce qui pourrait expliquer l'attribution à Alexandre Neckam ${ }^{45}$.

\section{Conclusion}

Les données encyclopédiques sont indéniablement nombreuses dans les sermons et ont été recherchées par les prédicateurs. Cependant, il est souvent difficile d'identifier les sources utilisées. Soit les citations sont imprécises, notamment dans le cas des textes reportés, dont l'exactitude dépend de la rigueur et de l'intérêt du reportateur. Soit les citations, plus explicites dans le cas des sermons rédigés, sont plus précises mais elles restent difficiles à identifier de façon littérale. Dans le cas du monde animal, utilisé par les prédicateurs des XIII et $\mathrm{XIV}^{\mathrm{e}}$ siècles, le Liber de natura rerum de Thomas de Cantimpré, dont la première rédaction semble dater de la décennie 1240 , semble avoir connu un succès certain auprès des prédicateurs. Cet usage était du reste explicitement souhaité dans son prologue.

Les données encyclopédiques sont ensuite exploitées de plusieurs manières. Souvent la faune occidentale est invoquée pour être interprétée allégoriquement ou moralisée. Plus rarement, comme dans le cas du sermon d'Eustache d'Arras, les propriétés de l'animal ou du végétal sont énumérées, exploitées, confirmées par des autorités bibliques, afin de construire toute une partie du développement du texte. On retrouve du reste cet usage structurant dans un sermon de Carême de Jacques de Voragine, qui élabore son plan autour des propriétés du loup ou de celles du fruit du cèdre ${ }^{46}$. s'appliquer aux sermons latins, mais aussi à ceux prêchés en langue romane, comme l'a 
souligné Michel Zink ${ }^{47}$. Cette vulgarisation est probablement passée par d'autres intermédiaires. Il faudrait désormais examiner plus en détail certains recueils classant alphabétiquement les naturalia et les reliant à leurs propriétés. Ces «recueils de propriétés » forment un genre assez proche des recueils de distinctions. Très peu étudiés et mal identifiés jusqu'ici, ils indiquent brièvement une propriété avant de la diviser en trois ou quatre interprétations utilisables par le prédicateur. C'est le cas par exemple de l' Angelus purus natura ou du Proprietates quarumdam rerum commençant par abies, qu'étudie Emmanuelle Kuhry ${ }^{48}$.

\section{BIBLIOGRAPHIE}

\section{Editions}

BARTHOLOMEUS ANGLICUS, De proprietatibus rerum, Frankfurt, 1601.

BARTHOLOMEUS ANGLICUS, De proprietatibus rerum I, prohemium, libri I-IV, VAN DEN ABEELE Baudouin, MEYER Heinz et alii (éd.), Turnhout, 2007, vol I.

CESARIUS HEISTERBACENSIS, Homiliae de infantia Servatoris, éd. COPPENSTEIN Johann Andreas (éd.), 1615, URL http://gahom.huma-num.fr/thema/index.php?id=14330\&lg=fr.

Glossa ordinaria, In Canticum Canticorum, Dove Mary (éd.), Turnhout, 1997, (Corpus Christianorum, Continuatio mediaevalis, 170).

JACQUES DE VORAGINE, Sermones aurei, CLUTIUS (éd.), 1760.

THOMAS CANTIMPRANTENSIS, Liber de natura rerum, BOESE Helmut (éd.), Berlin-New-York, 1973.

THOMAS DE СНовHAм, Summa de arte praedicandi, MORENZONI Franco (éd.), Turnhout, 1999.

[VINCENTIUS BELlovaCENSIS, Speculum maius]: Vincentii Burgundi Speculum quadruplex, naturale, doctrinale, morale, historiale. [...] Opera et studio Theologorum Benedictorum Collegii Vedastini in alma Academia Duacensi, Douai, I-IV, 1624 (éd. anast. Graz, 1961/65).

\section{Travaux}

BATAILLON Louis Jacques, «Les instruments de travail des prédicateurs au XIII ${ }^{e}$ siècle », in HASENOHR Geneviève, LONGÈRE Jean (dir.), Culture et travail intellectuel dans l'Occident médiéval, Paris, 1981, p. 197-209.

BATAILLON Louis Jacques, « Sermons rédigés, sermons reportés », Medioevo e rinascimento, 3, 1989, p. 69-86.

BATAILLON Louis Jacques, La prédication au XIII ${ }^{e}$ siècle en France et en Italie. Etudes et documents, Aldershot, 1993. 
BERLIOZ Marie-Anne, POLO DE BEAULIEU Jacques, « Les recueils d'exempla et la diffusion de l'encyclopédisme médiéval », in PICONE Michelangelo (dir.), L'enciclopedismo medievale, actes du colloque de San Giminiano, 6-10 octobre 1992, Ravenna, 1994, p. 179-212.

BINKLEY Peter, " Preachers' Responses to the Thirteenth-Century Encyclopaedism ", in BINCKLEY, P. (éd.), Pre-modern Encyclopaedic Texts. Proceedings of the second COMERS Congress, Groningen, 1-4 July 1996, Leiden, New York, Köln, 1997, p. 75-88.

BOYER Christine, Les sermons de Guillaume de Sauqueville : l'activité d'un prédicateur dominicain à la fin du règne de Philippe le Bel, Thèse de l'Université Lyon 2, 2007.

BOYER Christine, DELMAS Sophie, « Modèles de prédication pour modèle de gouvernement ? Le cas des sermons ad status au XIII ${ }^{\mathrm{e}}$ siècle", in Apprendre, produire, se conduire : le modèle au Moyen Âge,

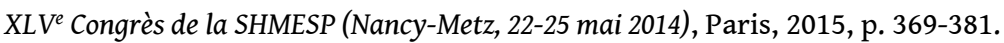

BURGHART Marjorie, Remploi textuel, invention et art de la mémoire : les Sermones ad status $d u$ Franciscain Guibert de Tournai (†1284), Thèse de l’Université Lyon 2, 2013.

CHARLAND Thomas Marie, Artes predicandi. Contribution à l'histoire de la rhétorique au Moyen Âge, Paris-Ottawa, 1936.

DELMAS Sophie, Un franciscain à Paris au milieu du XIII e siècle. Le maître en théologie Eustache d'Arras, Paris, 2010.

DRAELANTS Isabelle, «Scala mundi, scala celi de la A a la Z : claves para la comprensión de la obra universal de Juan Gil de Zamora. Exégesis, libri authentici y mediadores », Studia Zamorensia, 13, 2014, p. 27-70.

DRAELANTS Isabelle, « Modèles épistémologiques de l'enquête encyclopédique sur la nature des choses ob posteritatis utilitatem » in Apprendre, produire, se conduire : le modèle au Moyen Âge, XLV Congrès de la SHMESP (Nancy-Metz, 22-25 mai 2014), Paris, 2015, p. 235-260.

FALMAGNE Thomas, « Les instruments de travail d'un prédicateur cistercien. A propos de Jean de Villers », in HAMESSE Jacqueline, HERMAND Xavier (éd.), De l'homélie au sermon, histoire de la prédication médiévale. Actes du colloque international de Louvain-La-Neuve (9-11 juillet 1992), Louvainla-Neuve, 1993 (Publications de l'Institut d'études médiévales, Textes, Études, Congrès, 14), p. 183-239.

FRIEDMAN John Block, « Peacocks and Preachers : Analytic Technique in Marcus of Orvieto's Liber de moralitatibus, Vatican lat. MS 5933 », in Beasts and Birds of the Middle Ages. The Bestiary and its Legacy, CLARK Willene, MCMUNN Meradith (éd.), Philadelphia, 1989, p. 179-196.

HERBERT John Alexander, Catalogue of Romances in the Department of Manuscripts in the British Museum, London, 1910, vol. 3.

KUHRY Emmanuelle, «Dictionnaires, distinctions, recueils de propriétés en milieu cistercien : outils pour la prédication, sources pour l'étude de la nature ", in TURCAN-VERKERK Anne-Marie, GANDIL Pierre, STUTZMAnN Dominique, FALMAGne Thomas (éd.), Les Cisterciens et la transmission des textes (XII ${ }^{e}$-XVIII ${ }^{e}$ siècles), actes du colloque international de Troyes, 22-24 novembre 2012, Paris-Turnhout, 2017.

LUSIGNAN Serge, Préface au Speculum Maius de Vincent de Beauvais : réfraction et diffraction, MontréalParis, 1979, (Cahiers d'études médiévales).

MASSON Xavier, Une voix dominicaine dans la cité. Le comportement exemplaire du chrétien dans l'Italie du Trecento d'après le recueil de sermons de Nicoluccio di Ascoli, Rennes, 2009. 
MEIER Christel, « Organisation of Knoweldge and Encyclopaedic Ordo: Functions and Purposes of a Universal Literary Genre ", in BINKLEY Peter (éd), Pre-modern encyclopaedic texts. Proceedings of the second COMERS Congress, Groningen, 1-4 july 1996, Leiden, 1997, p. 103-126.

MEYER Heinz, « Die Predigerenzyklopädie. Textsorte und Gebrauch unter methodischen Aspekten ", in KELLER Hagen, MEIER Christel, sUNTRUP Rudolf (éd.), Pragmatische Dimensionen mittelalterlicher Schriftkultur. Akten des Internationalen Kolloquiums 26.-29. Mai 1999, München, 2002, (Münstersche Mittelalterschriften, 79), p. 177-190.

MORENZONI Franco, Des écoles aux paroisses. Thomas de Chobham et la promotion de la prédication au début du XIII siècle, Paris, 1995.

MORENZONI Franco, «Les animaux exemplaires dans les recueils de Distinctiones bibliques alphabétiques du XIII ${ }^{e}$ siècle ", in L'animal exemplaire au Moyen Âge. $V^{e}-X V^{e}$ siècle, BERLIOZ MarieAnne, POLO DE BEAULIEU Jacques (dir.), Rennes, 1999, p. 171-190.

MURPHY James Jerome, Rhetoric in the Middle Ages. A History of Rhetorical Theory from St Augustine to the Renaissance, Berkeley-Los Angeles-London, 1975.

NADEAU Alain, « Faire œuvre utile. Notes sur le vocabulaire de quelques prologues dominicains du XIII ${ }^{\mathrm{e}}$ siècle » in LUSIGNAN Serge, PAULMIER-FOUCART Monique, DUCHENNE Marie-Christine (dir.), Lector et compilator. Vincent de Beauvais, frère prêcheur. Un intellectuel et son milieu au XIII ${ }^{e}$ siècle. Actes du Colloque de Royaumont des 9-11 juin 1995, Grâne, 1997, (Rencontres à Royaumont, 9), p. 77-96. PAUlmier-FoucARt Monique, DUChenne, Marie-Christine (collab.), Vincent de Beauvais et le Grand Miroir du monde, Turnhout, 2004, (Témoins de notre histoire).

TALAVERA ESTESO Francisco José, « Ciencia y predicación en el siglo XIII : el Liber de natura rerum de Tomás de Cantimpré », Ciencia tomística, 104,1977, p. 407-450.

TUBACH Frederic C., Index exemplorum: A Handbook of Medieval Religious Tales, Helsinki, 1969.

VAN DEN ABEELE Baudouin, "L'allégorie animale dans les encyclopédies latines du Moyen Âge », in L'animal exemplaire au Moyen Âge. $V^{e}-X V^{e}$ siècle, BERLIOZ Marie-Anne, POLO DE BEAULIEU Jacques (dir.), Rennes, 1999, p. 123-143.

VAN DEN ABEELE Baudouin, «Diffusion et avatars d'une encyclopédie : le Liber de natura rerum de Thomas de Cantimpré », in DE CALLATAŸ Godefroid, VAN DEN ABEELE Baudouin (dir.), Une lumière venue d'ailleurs. Héritages et ouvertures dans les encyclopédies d'Orient et d'Occident au Moyen Age, Louvain-la-Neuve, 2008 (Réminisciences, 9), p. 141-176.

VAN DEN ABEELE Baudouin, «Les stratégies olfactives des animaux et leur moralisation », in PARAVICINI BAgliani Agostino (éd.), Parfums et odeurs au Moyen Age. Science, usage, symboles, Firenze, 2015, (Micrologus' Library, 67), p. 429-444.

VOISENET Jacques, Bestiaire chrétien. L'imagerie animale des auteurs du Haut Moyen Âge ( $V^{e}-X I^{e}$ siècle), Toulouse, 1994.

ZINK Michel, La prédication en langue romane avant 1300, Paris, 1976.

\section{NOTES}

1. Je remercie vivement Isabelle Draelants pour sa patience et ses précieuses suggestions. 
BINKLEY P., « Preachers' Responses to the Thirteenth-Century Encyclopaedism », in Binckley, P. (éd.), Pre-modern Encyclopaedic Texts. Proceedings of the second COMERS Congress, Groningen, 1-4 July 1996, Leiden, New York, Köln, 1997, p. 75-88 : 88.

2. BATAILLON L. J., La prédication au XIII ${ }^{e}$ siècle en France et en Italie. Etudes et documents, Aldershot, 1993, IV : 199 pour cette citation. Voir aussi p. 197-209.

3. BERLIOZ M.-A., polo de BEAUlieu J., «Les recueils d'exempla et la diffusion de l'encyclopédisme médiéval », in PICONE Michelangelo (dir.), L'enciclopedismo medievale, actes du colloque de San Giminiano, 6-10 octobre 1992, Ravenna, 1994, p. 179-212.

4. Sur cette utilité, voir en dernier lieu DRAELANTS I., " Modèles épistémologiques de l'enquête encyclopédique sur la nature des choses ob posteritatis utilitatem " in Apprendre, produire, se conduire : le modèle au Moyen Âge, XLV Congrès de la SHMESP (Nancy-Metz, 22-25 mai 2014), Paris, 2015, p. 235-260, dont sont aussi tirées les citations ci-dessous et leurs références.

5. BARTHolomeus anglicus, De proprietatibus rerum I, prohemium, libri I-IV : 51. Sur le concept d' utilitas dans les prologues, voir NADEAU A., «Faire œuvre utile. Notes sur le vocabulaire de quelques prologues dominicains du XIII ${ }^{\mathrm{e}}$ siècle » in LUSIGNAN S., PAULMIER-FOUCART M., DUCHENNE M.-C. (dir.), Lector et compilator. Vincent de Beauvais, frère prêcheur. Un intellectuel et son milieu au XIII siècle. Actes du Colloque de Royaumont des 9-11 juin 1995, Grâne, 1997 " Rencontres à Royaumont, 9 ", p. 77-96.

6. LUSIGNAN S., Préface au Speculum Maius de Vincent de Beauvais : réfraction et diffraction, MontréalParis, 1979, (Cahiers d'études médiévales) : 118. Pour la traduction du Libellus apologeticus, cf. PAULMIER-FOUCART M., De utilitate operis et apologia actoris [utilité de l'œuvre et apologie de l'auteur], in Vincent de Beauvais et le Grand Miroir du monde, Turnhout, 2004 (Témoins de notre histoire) : 152.

7. Cité par BERLIOZ, POLO DE BEAULIEU, «Les recueils d'exempla...»: 182 et note 11. Voir aussi TALAVERA ESTESO F. J., «Ciencia y predicación en el siglo XIII : el Liber de natura rerum de Tomás de Cantimpré », Ciencia tomística, 104, 1977, p. 407-450 : 415-416.

8. Ce passage traduit est cité par VAN DEN ABEELE B., "L'allégorie animale dans les encyclopédies latines du Moyen Âge ", in L'animal exemplaire au Moyen Âge. $V^{e}-X V^{e}$ siècle, BERLIOZ M.-A., POLO DE BEAULIEU J. (dir.), Rennes, 1999, p. 123-143 : 131. Voir aussi la synthèse proposée par VAN DEN ABEELE B., «Diffusion et avatars d'une encyclopédie : le Liber de natura rerum de Thomas de Cantimpré ", in DE CALLATAŸ G., VAN DEN ABEELE B. (dir.), Une lumière venue d'ailleurs. Héritages et ouvertures dans les encyclopédies d'Orient et d'Occident au Moyen Age, Louvain-la-Neuve, 2008, p. 141-176.

9. Voir en dernier lieu VAN DEN ABEELE B., «Les stratégies olfactives des animaux et leur moralisation ", in PARAVicini BAGLIANi A. (éd.), Parfums et odeurs au Moyen Age. Science, usage, symboles, Firenze, 2015, « Micrologus' Library, 67 », p. 429-444 et la bibliographie.

10. Pour ces trois exemples, voir BERLIOZ, POLO DE BEAULIEU, « Les recueils d'exempla... » : 186-189.

11. TALAVERA ESTESO, « Ciencia y predicación en el siglo XII... » : 417.

12. BARTHOLOMEUS ANGLICUS, DPR, I, prohemium : 10-11.

13. Voir FRIEDMAN J. B., «Peacocks and Preachers : Analytic Technique in Marcus of Orvieto's Liber de moralitatibus, Vatican lat. MS $5933 »$, in Beasts and Birds of the Middle Ages. The Bestiary and its Legacy, CLARK W., MCMUNN M. (éd.), Philadelphia, 1989, p. 179-196.

14. MEIER C., «Organisation of Knoweldge and Encyclopaedic Ordo: Functions and Purposes of a Universal Literary Genre ", in BINKLEY P. (éd), Pre-modern encyclopaedic texts. Proceedings of the second COMERS Congress, Groningen, 1-4 july 1996, Leiden, 1997, p. 103-126 : 118-199 ; MEYER H., « Die Predigerenzyklopädie. Textsorte und Gebrauch unter methodischen Aspekten », in KELLER H., MEIER C., SUNTRUP R. (éd.), Pragmatische Dimensionen mittelalterlicher Schriftkultur. Akten des 
Internationalen Kolloquiums 26.-29. Mai 1999, München, 2002, « Münstersche Mittelalterschriften, 79 », p. 177-190.

15. Sur les Artes predicandi, voir notamment CHARLAND T. M., Artes predicandi. Contribution à l'histoire de la rhétorique au Moyen Âge, Paris-Ottawa, 1936 ; MURPHY J. J., Rhetoric in the Middle Ages. A History of Rhetorical Theory from St Augustine to the Renaissance, Berkeley-Los Angeles-London, 1975 ; M BRISCOE M. G., Artes predicandi, Turnhout, 1992, «Typologie des sources du Moyen Âge occidental, $61 »$; MORENZONI Franco, Des écoles aux paroisses. Thomas de Chobham et la promotion de la prédication au début du XIII ${ }^{e}$ siècle, Paris, 1995 : 189-240.

16. MORENZONI, Des écoles aux paroisses, $1999: 195$.

17. MORENZONI, Des écoles aux paroisses, 1999 : 213, 218. Voir aussi MORENZONI F., "Les animaux exemplaires dans les recueils de Distinctiones bibliques alphabétiques du XIII ${ }^{\mathrm{e}}$ siècle ", in L'animal exemplaire au Moyen Âge. $V^{e}-X V^{e}$ siècle, BERLIOZ M.-A., POLO DE BEAULIEU J. (dir.), Rennes, 1999, p. $171-190: 171-172$.

18. тномAS De сновнам, Summa de arte praedicandi, c. 7, 1.661: Et Dominus ideo dicitur leo quia dominatur super omnes creaturas. / Virtus autem leonis in pectore est, firmitas in capite. / Capti leones timent strepitum rotarum. / Super omnia timet gallum album. Ces exemples sont monnaie courante dans les encyclopédies : cf. entre autres DRAELANTS I., "Scala mundi, scala celi de la A a la Z : claves para la comprensión de la obra universal de Juan Gil de Zamora. Exégesis, libri authentici y mediadores », Studia Zamorensia, 13, $2014:$ 27-70.

19. BARTHOLOMEUS ANGLICUS, De proprietatibus rerum, XVIII, c. $63: 1081$.

20. MORENZONI, Des écoles aux paroisses, 1999 : 227.

21. Ce passage est cité par MASSON X., Une voix dominicaine dans la cité. Le comportement exemplaire du chrétien dans l'Italie du Trecento d'après le recueil de sermons de Nicoluccio di Ascoli, Rennes, 2009 p. 184.

22. CHARLAND, Artes predicandi, $1936: 204,396$.

23. TALAVERA ESTESO, « Ciencia y predicación en el siglo XII... », p. 416-417.

24. Pour ce passage de saint Augustin voir DRAELANTS, « Modèles épistémologiques... » : 243-244.

25. BATAILLON L. J., « Sermons rédigés, sermons reportés (XII ${ }^{\mathrm{e}}$ siècle) », Medioevo e rinascimento, 3 , 1989, p. 69-86.

26. Voir le catalogue des œuvres d'Eustache dans DELMAS S., Un franciscain à Paris au milieu du XIII siècle. Le maître en théologie Eustache d'Arras, Paris, 2010 : 347-404. Ces sermons sont sur le point d'être édités dans le Corpus Christianorum, Continuatio Mediaevalis.

27. vOISENET J., Bestiaire chrétien. L'imagerie animale des auteurs du Haut Moyen Âge ( $V^{e}-X I^{e}$ siècle), Toulouse, 1994, p. 69 et 276.

28. KUHRY E., "Dictionnaires, distinctions, recueils de propriétés en milieu cistercien: outils pour la prédication, sources pour l'étude de la nature ", in TURCAN-VERKERK A.-M., GANDIL P., STUTZMANN D., FALMAGne T. (éd.), Les Cisterciens et la transmission des textes (XII ${ }^{e}$-XVIII ${ }^{e}$ siècles), actes du colloque international de Troyes, 22-24 novembre 2012, Paris-Turnhout, 2017.

29. Glossa ordinaria, In Canticum Canticorum : 131.

30. CESARIUS HEISTERBACENSIS, Homiliae de infantia Servatoris : $153 \mathrm{~b}$.

31. C'est le cas dans le sermon 89 de Guillaume de Sauqueville. Exemplo naturali hoc apparet nam legitur de aquila quod propter magnam delectationem quam habet claritatem solis intuendo, nidum suum fabricat et construit super arborem altiorem et oculos dirigit ad solem, ut claritatem solis limpidius contempletur dans l'annexe 5 de la thèse de BOYER C., Les sermons de Guillaume de Sauqueville: l'activité d'un prédicateur dominicain à la fin du règne de Philippe le Bel, thèse de l'Université Lyon 2, 2007. Cf. aussi тUвACH F. C., Index exemplorum : a handbook of medieval religious tales, Helsinki, 1969, $\mathrm{n}^{\circ}$ 1837. HERBERT J. A., Catalogue of romances in the Department of manuscripts in the British Museum, vol. 3, Londres, 1910 : H69 n 108, H378 nº 81. Jacques de Voragine, Sermones aurei : 48a.

32. BATAILLON, « Sermons rédigés, sermons reportés ». 
33. BOYER C., DELMAS S., "Modèles de prédication pour modèle de gouvernement ? Le cas des sermons ad status au XIII ${ }^{\mathrm{e}}$ siècle", in Apprendre, produire, se conduire : Le modèle au Moyen Âge, XLV Congrès de la SHMESP (Nancy-Metz, 22-25 mai 2014), Paris, 2015, p. 369-381.

34. BURGHART M., Remploi textuel, invention et art de la mémoire : les Sermones ad status du Franciscain Guibert de Tournai (†1284), thèse de l'Université Lyon 2, 2013. Elle m'a généreusement permis de consulter et d'utiliser son travail. Qu'elle en soit chaleureusement remerciée ici.

35. BURGHART, Remploi textuel, 2013 :vol. I, 123.

36. Formicoleon ideo sic vocatur, quia formicarum est leo vel quia formica pariter et leo. Est enim animal parvum, formicis satis infestum, quod in pulvere se abscondit et formicas frumenta gestantes interficit. Proinde autem formica et leo vocatur, quia aliis animalibus est ut formica, formicis autem ut leo. VINCENT DE BEAUVAIS, Speculum naturale, XX, c. 135, col. 1358.

37. Formice autem, ut in libris naturalium legitur, ducem non habent, nulla eas ducente, ne cecus cecum ducat. Sunt enim cecutientes nec clare uidentes, unde et pro baculo datum est eis quoddam additamentum in parte anteriori quo se dirigant uiam pertemptando, quia qui intelligentes et discreti non sunt, non debent alios regere, sed sufficiat eis si se possunt regere. BURGHART, Remploi textuel, vol. I : 492.

38. FALMAGNE T., «Les instruments de travail d'un prédicateur cistercien. À propos de Jean de Villers ", in HAMESSE J., HERMAND X. (éd.), De l'homélie au sermon, histoire de la prédication médiévale. Actes du colloque international de Louvain-La-Neuve (9-11 juillet 1992), Louvain-la-Neuve, 1993, p. 183-239: 217.

39. MASSON, Une voix dominicaine, 2009: 212-213 et note 127 pour la référence à Vincent de Beauvais. Ajoutons que chez Vincent de Beauvais (Speculum naturale, XIII, c. 2), le passage n'a pas comme sources "Alexander», mais Dioscoride. Il pourrait s'agir peut-être du speculum speculationum d'Alexandre Neckam.

40. MASSON, Une voix dominicaine, 2009 : 219-220 et note 158. Ce titre pourrait également faire songer au Liber rerum auquel renvoie par exemple de Thomas de Cantimpré. Je n'ai malheureusement pas pu consulter le texte latin de ces sermons pour faire des vérifications.

41. Je remercie vivement I. Draelants qui m'a aidée à faire ces vérifications. Il n'est pas à exclure que le prédicateur ait eu à sa disposition une version différente de celle que nous connaissons. Par ailleurs, il est frappant de constater que ce passage renvoie au serpent et à son interprétation symbolique : le serpent est justement pris en exemple par saint Augustin dans le passage du De doctrina christiana évoqué ci-dessus, note 24.

42. Ce paragraphe doit beaucoup à l'article de MORENZONI, «Les animaux exemplaires dans les recueils de Distinctiones ", $1999: 180$.

43. Je n'ai pas eu accès au manuscrit d'Avignon qu'a vu Morenzoni ; cette citation est tirée de son article « Les animaux exemplaires ».

44. Voir thomas cantimprantensis, Liber de natura rerum, BOESE H. (éd.), Berlin-New-York, 1973 (consulté ici sur le manuscrit London, British Library, Harley 3717, f. 60rb.)

45. VAN DEN ABEELE, « Diffusion et avatars d'une encyclopédie » : 146.

46. Dicit tamen Chrysostomus super Mattheum quod lupus si tegatur pelle ouina, potest tamen cognosci quod sit lupus per tria. Primo per actum, quia lupus dentibus mordet; ouis autem non habet dentes, unde mordeat. (...) Secundo per ululatum, quia lupus uersus celum ululat; ouis autem uersus terram ad ipsam balat. (...) Tertio per cibum, quia lupus cibatur carnibus ; ouis uero herbis uirentibus pascitur. http:// sermones.net/thesaurus/document.php?id=jvor_266\&list=dis\&dis=dis_3\#dis_3.

Un autre sermon de Carême est construit à partir des propriétés du fruit du cèdre : Poma enim cedrina in aliqua sui parte sunt dulcia (...) in aliqua sunt acetosa (...) in alia inter dulce et acetosum sunt media, per que signantur in Christo tres substantie. http://sermones.net/thesaurus/document.php? id=jvor_203\&list=dis\&dis=dis_2\#dis_2

47. zINK Michel, La prédication en langue romane avant 1300, Paris, 1976, p. 358-363. 
48. Ses premières découvertes sur ces deux recueils sont présentes dans sa thèse d'histoire médiévale de l'Université de Lorraine soutenue en janvier 2014 : La Compilatio de libris naturalibus Aristotelis et aliorum quorundam philosophorum ou Compendium philosophie. Histoire et édition préliminaire partielle d'une compilation philosophique du XIII ${ }^{e}$ siècle. Sur la question des recueils de propriétés naturelles, son article est à paraître: KUHRY, «Dictionnaires, distinctions, recueils de propriétés en milieu cistercien ».

\section{RÉSUMÉS}

Les auteurs des encyclopédies médiévales veulent être utiles aux prédicateurs : les prologues, les moralisations accompagnant les informations sur la nature le montrent. De même, les prédicateurs font allusion aux encyclopédies dans les Artes predicandi, dans les distinctions ou dans leurs sermons (animaux, plantes). Cet article en présente quelques exemples.

Medieval encyclopaedia aim at providing tools for the preachers, as suggest their prologues and some moralizations regarding naturalistic information. Similarly, the preachers refer to encyclopaedias in their Artes predicandi, in distinctiones, in sermons, about the properties of animals or plants. This articles aims at showing some examples.

\section{INDEX}

Mots-clés : Sermons, encyclopédies, artes predicandi, distinctions, moralisation

Keywords : Sermons, encyclopedias, artes predicandi, distinctions, moralization

\section{AUTEUR}

\section{SOPHIE DELMAS}

Sophie Delmas est docteur en histoire, enseignante et membre associée à une équipe CNRS. Spécialisée dans l'étude des ordres mendiants, elle a publié sa thèse sur Un franciscain à Paris. Le maître en théologie Eustache d'Arras (o.f.m.) au milieu du XIIIe siècle, Paris, 2010. Membre du comité de rédaction d'Etudes franciscaines, ses recherches et ses articles portent sur l'histoire politique, religieuse et intellectuelle des XIIe-XVe siècles. Saint Antoine de Padoue est sorti de presse chez Plon en 2017 et De Louis IX à Saint Louis. Généalogie d'un mythe, chez Ellipses, est sous presse. 